\title{
Le mouvement des écrivains finno-ougriens
}

Finno-Ugric kinship in Komi literature: common heritage or recent influence?

Soome-ugri sugulus komi kirjanduse : kas ühine pärand või hiljutised mõjud?

\section{Eva Toulouze}

\section{OpenEdition}

\section{Journals}

Édition électronique

URL : https://journals.openedition.org/efo/3678

DOI : $10.4000 /$ efo.3678

ISSN : 2275-1947

\section{Éditeur}

INALCO

\section{Édition imprimée}

Date de publication : 1 janvier 2014

ISBN : 978-2-343-05394-3

ISSN : 0071-2051

\section{Référence électronique}

Eva Toulouze, "Le mouvement des écrivains finno-ougriens », Études finno-ougriennes [En ligne], 46

2014, mis en ligne le 13 octobre 2015, consulté le 08 juillet 2021. URL : http://

journals.openedition.org/efo/3678; DOI : https://doi.org/10.4000/efo.3678

Ce document a été généré automatiquement le 8 juillet 2021.

\section{(c) (7) (8)}

Études finno-ougriennes est mis à disposition selon les termes de la Licence Creative Commons Attribution - Pas d'Utilisation Commerciale 4.0 International. 


\section{Le mouvement des écrivains finno- ougriens}

Finno-Ugric kinship in Komi literature: common heritage or recent influence?

Soome-ugri sugulus komi kirjanduse : kas ühine pärand või hiljutised mõjud?

\section{Eva Toulouze}

1 Dans cet article, je m'appuie surtout sur mon expérience personnelle: en effet, à l'exception du premier Congrès des écrivains finno-ougriens en 1989, j'ai été associée à tous les autres et je fais partie depuis 1996 de la direction de l'Association des Littératures finno-ougriennes.

\section{Le rôle des écrivains dans la promotion des identités nationales finno-ougriennes}

2 Les identités nationales ne sont pas atemporelles: elles sont inscrites dans l'espace mais également dans le temps. Pour les communautés finno-ougriennes de Russie, l'époque de l'émergence des identités nationales correspond, dans la plupart des cas, à celle où une intelligentsia naît des tentatives missionnaires de scolarisation. Pour ces communautés, c'est en effet la première occasion où elles doivent s'identifier entre autres autour d'une langue.

3 L'autoidentification en soi n'était bien sûr pas quelque chose de nouveau. Avant le $\mathrm{XIX}^{\mathrm{e}}$ siècle, les personnes avaient été amenées à s'identifier, certes, dans le contexte administratif dans lequel s'effectuait la rencontre avec l'autre, fût-il tatar ou russe, détenteur d'un pouvoir étatique. Mais dans lesdites relations, la catégorisation ethnique n'était sans doute pas particulièrement d'actualité. Pour l'impôt, c'est l'individu et le groupe qui comptaient, plus que l'ethnie. La perspective classificatoire devient de plus en plus prégnante au fur et à mesure que le siècle avance ${ }^{1}$, pour arriver au recensement de 1889, qui n'est pas par hasard le premier tenu dans l'Empire, et où la « nationalité » est structurante. 
Pour les Finno-ougriens, au XIX ${ }^{e}$ siècle, l'identification ethnique n'était pas signifiante : ces groupes n'avaient pas d'élite unificatrice. Populations sédentaires, attachées à la terre qu'elles cultivaient, ces communautés ne s'étaient jamais rassemblées, unifiées, délimitées. Le besoin ne s'en était pas fait sentir ${ }^{2}$. Cela ne veut pas dire qu'il n'y ait pas eu dans l'histoire des moments critiques où cette identification s'était imposée, par exemple dans le cadre d'affrontements avec des ennemis ou de soulèvements. Encore n'avons-nous aucun témoignage direct. Nous ne disposons que du regard identificateur de l'autre (guerres tchérémisses, information sur la participation d'Oudmourtes et de Maris aux soulèvements de Sten'ka Razin et Pugačëv). Sans doute les tensions entre Maris des plaines et Maris des collines dans les années qui ont suivi la conquête par les troupes d'Ivan le terrible des territoires du khanat de Kazan' ont-elles été un élément cristallisateur de leurs identités définies par contraste (Kappeler 1982, p. 90, Istorija 1987 I, p. 18, Nolde 1953, p. 56, Marist 1992, Griškina 1994a, p. 128-131).

Mais dès lors qu'émerge le projet, d'origine missionnaire, d'alphabétiser ces communautés conformément à leur langue vernaculaire, un élément d'identification précis apparaît dans le quotidien - la langue. Loin d'apparaître comme un trait d'identification négatif, la langue vernaculaire, distinguant les autochtones des Russes, devient un même un élément d'intégration: dans la stratégie d'Il'minskij, en effet, l'usage des langues vernaculaires a pour objectif essentiel, à terme, son propre dépassement et l'adoption totale du christianisme, donc de la russitude, donc de la langue russe. Le père de l'école pour autochtones reconnaît lui-même son rôle potentiel dans ce passage clairvoyant :

Nous sommes devant un dilemme : si, par peur de chacune des nationalités, nous ne faisons pas de place aux langues allogènes à l'école et à l'église à un niveau suffisant pour garantir une assimilation solide, complète et convaincue de la foi chrétienne, tous les allogènes se fondront en une seule tribu, unie par la langue (tatare) et par la foi (musulmane). Si nous faisons appel aux langues locales, et même si nous soutenons chacune des nationalités, ce seront des nationalités diverses, minuscules, sans rapport avec le monde tatar et unies au peuple russe par la même foi. Choisissez ! (Il'minskij 1898, pp. 398-399).

6 Dans cette déclaration, Il'minskij présente l'identité tatare comme assurée, au même titre que l'identité russe, voir comme une identité extensible. Mais les autres identités de la région sont encore en devenir. En même temps, le missionnaire russe ne les voit pas comme menaçantes ou potentiellement dangereuses pour la domination russe. En fait, le scénario qu'il présente ici correspond à celui qui s'est effectivement réalisé.

7 Mais si l'existence et le mode de structuration du système Il'minskij, basé sur les langues vernaculaires, ont certainement, à la fin du XIX siècle, permis de consolider des identités liant un nom et une langue, leur affirmation est parachevée quand, dans la première décennie $\mathrm{du} \mathrm{xx}^{\mathrm{e}}$ siècle puis avec la Révolution d'octobre, ceux qui disposent désormais de cet outil de modernité qu'est une langue écrite prennent leur autonomie et s'en servent dans des buts qu'Il'minskij ne pouvait pas seulement imaginer. Ils s'en servent, pour l'essentiel, par l'écriture créatrice (Toulouze 2001).

8 Chez les paysans, le fait d'avoir un pope qui prêche dans leur langue, d'envoyer leurs enfants à une école et pas à une autre, sont autant d'éléments qui leur font sentir une appartenance à une communauté qui dépasse le village. Mais cette perception est surtout réelle chez ceux qui vont bientôt entrer dans l'action, cette nouvelle élite, petit groupe d'intellectuels, qui sentent qu'il en va de leur responsabilité de permettre l'affirmation d'un groupe, leur peuple. 
Dans leurs écrits, ces intellectuels proclament la nouveauté de leur vision, et donnent à la nouvelle identité, présentée comme ancestrale, ses lettres de noblesse. Sergej Čavajn, dans son premier poème en langue marie, ne proclame pas ouvertement les vertus du peuple mari, mais il le fait très tôt en prose avec l'histoire ancienne des Maris : Sur le peuple mari ${ }^{3}$ a été écrit en 1908 et publié dans l'un des Almanachs maris ${ }^{4}$. De plus, dans des récits (Jylanda 1912) et des poèmes, il se concentre sur les héros maris ayant lutté contre les Russes. Avant lui, les auteurs maris de manuels, à leur manière, avaient commencé à poser cette identité comme allant de soi : rédiger des manuels revient en effet à poser le caractère central de la langue vernaculaire dans la construction de la personnalité d'un enfant. M. Evsev'ev (1864-1931), avec La noce mordve (1892), prend appui sur les traditions, imposant la notion - depuis fort discutée - de Mordve; Grigorij Vereščagin, avec ses écrits ethnographiques, fait de même. Chez les Oudmourtes, cette notion atteint sa forme achevée dans les écrits de K. Gerd avant la Révolution d'octobre: le peuple oudmourte, les Oudmourtes, y sont l'un des personnages les plus chéris du poète. Chez les Komis, cette identité se manifeste, chez leur premier acteur social de la komitude, par la prise en compte de l'histoire, et ce dans le dialogue avec l'autre: Stéphane de Permie, qui donne au komi son premier alphabet, sa première notation, est en fait un héros russe. Mais c'est autant dans la prise en charge du passé chrétien que dans l'affirmation du komi en tant que langue de la nouvelle religion que Stéphane et, dans son hagiographie, Georgij Lytkin, donnent à la communauté des Komis ses lettres de noblesse. Je pourrais sans doute citer Ivan Kuratov, ce poète solitaire et inconnu, qui dès le milieu du XIX siècle proclamait l'amour de la langue et du pays komi. Mais Kuratov n'est qu'une hirondelle. Celui qui a un impact immédiat sur le paysage intellectuel, c'est Lytkin (Toulouze 2010, p.28-29).

10 Avec le soulèvement qui les propulse au pouvoir, les bolcheviks sont confrontés à une série de problèmes de grande ampleur, qu'ils abordent notamment par leurs premiers actes spectaculaires, quelques heures après le succès de l'insurrection : les trois décrets sur la paix, sur la terre et sur les nationalités. Les nationalités ne présentaient pas, pour eux, un problème de principe. Le modèle marxiste qui les guide ne voit l'ethnicité que comme un élément de superstructure semant le trouble dans l'analyse des véritables contradictions, celles de classe. Mais les bolcheviks, en Russie, sont confrontés à un problème concret: le mécontentement de nombreuses ethnies, qui plus est fort puissantes. Le décret vise à débarrasser les détenteurs du pouvoir d'un cercle de problèmes qu'ils ne sont pas en mesure d'aborder efficacement. Ces ethnies susceptibles de mettre en cause le nouveau pouvoir, ce ne sont certainement pas les petits groupes qui abondent en Russie; ce sont les Polonais, les Ukrainiens, les peuples baltiques. L'identité polonaise ne date pas d'hier, elle a près d'un siècle d'existence. Les peuples de la Baltique ne sont pas dangereux par leur nombre, mais par leur influence : Estoniens et Lettons, massivement alphabétisés depuis plus d'un siècle par le protestantisme, forment une partie non négligeable de l'élite pétersbourgeoise. Mais une fois que la déclaration est rendue publique, elle ne peut pas ne pas s'appliquer (pour autant qu'elle s'applique) aux autres communautés. Dans le chaos dominant, les élites s'en emparent. Ainsi, les actes des bolcheviks eux-mêmes viennent apporter de l'eau au moulin d'ethnies qui n'étaient, pour certaines, qu'en cours de formation.

11 Dans les communautés qui nous intéressent, ce sont ces instituteurs et ecclésiastiques instruits qui s'emparent des possibilités offertes par la conjonction des décrets bolcheviques et de la vacance effective du pouvoir. Comment les utiliser, cela fait 
l'objet d'innombrables réunions, congrès, conférences dans la région de la Volga entre 1918 et 1919. Les SR y font entendre leur voix : ils sont encore présents sur la scène politique ; les idées d'autonomie étatique, voire de fédération régionale (l'Idel-Oural, pour mentionner un terme qui n'a jamais eu de fortune chez les Finno-ougriens) s'y font entendre (Colarz 1956, p. 27, Revolucija 1930, p. 414-415). Les bolcheviks, organisés et opportunistes, tirent leur épingle du jeu : leurs propositions d'autonomie culturelle apparaissent comme la solution idéale qui fait l'unanimité de la jeune intelligentsia. La prééminence bolchevique s'explique par plusieurs raisons. Tout d'abord, les bolcheviks avaient un besoin cuisant d'alliés qu'ils vont aller chercher auprès des groupes mal aimés du régime précédent. Les "allogènes ", clairement, en sont. Ensuite, leur volonté d'hégémonie politique ne les dispose guère au compromis avec les revendications politiques des nationalités de la Russie. Or, les Finno-Ougriens n'ont guère de revendications politiques, leurs aspirations ne sont que culturelles, et donc peu dangereuses, et leurs élites n'ont guère l'ambition du pouvoir. Enfin Lénine lui-même, qui dans ces années est encore le principal décideur du POSDR(b), est originaire de la région de la Volga ; il tend une oreille bienveillante aux revendications des élites de ces peuples et est sensible au danger que leur fait courir le "chauvinisme grand-russe", qu'il connaissait bien et qu'il n'affectionnait guère.

Chez les Komis, dont l'identité s'était affirmée avant les autres, sans doute du fait de leurs relations anciennes avec le monde russe, le modèle suivi est un peu différent: dans une région où l'immense majorité de la population est komie, une élite non seulement culturelle, mais aussi politique, émerge dans ces années à proprement parler révolutionnaires. Suffisamment de Komis avaient des expériences de travail hors de leur terre, connaissaient l'usine, avaient rencontré fort tôt les bolcheviks : le moment venu, aguerris par une guerre civile fratricide, ils ont de quoi former un gouvernement de communistes komis. Mais ces communistes, pour être militants politiques, n'en sont pas moins komis et ils ont à cœur les intérêts de ce qu'ils ressentent comme étant leur peuple: ceci explique les particularités de la construction du socialisme dans cette région (Toulouze 2010, p. 30-35).

Dans le monde fennique, les enjeux sont différents : c'est que la Finlande est secouée par une guerre civile. La force d'attraction du camp communiste finlandais y est le facteur central. Avec l'échec des Rouges dans la guerre civile, beaucoup se réfugient dans la Carélie soviétique : la construction du socialisme s'y veut comme un modèle face à la Finlande indépendante et « bourgeoise » qui se met en place de l'autre côté de la frontière.

Dans les toundras européennes et en Sibérie occidentale, les autochtones sont totalement en dehors du monde politique russe. Seuls quelques individus sont alphabétisés. Pour les communautés, les affrontements entre Russes restent extérieurs (Karšakova 1996, p. 34, Vella 1991, p. 53). Les structures territoriales spécifiques portant les noms d'ethnies autochtones sont des constructions élaborées par le pouvoir russe à l'intention de communautés qui ne sont pas en mesure de participer directement au façonnement de leur avenir, même au moment où la situation politique était suffisamment ouverte pour permettre un semblant de dialogue.

C'est ainsi que, dans la région Oural-Volga, la force active derrière la mise en place des autonomies nationales - les oblast' nationales des années 1920-1921 - est l'intelligentsia. Elle fait tout. En Oudmourtie, l'un des premiers Oudmourtes communistes, Trofim Borisov, le premier président des Soviets, est médecin ; mais il est aussi l'auteur, 
en 1919, du premier recueil de poésie en oudmourte, rassemblant les œuvres de différents jeunes auteurs. Ces hommes (il n'y a pas de femmes parmi eux) organisent, militent, aménagent la langue, écrivent, jouent sur scène, enseignent, interviennent dans des congrès, étudient dans la capitale ou ailleurs dans le monde. Ils ont pratiquement carte blanche pendant quelques années. Ils ne sont pas nombreux, mais la responsabilité tombe sur eux ; c'est à eux de faire l'histoire.

Ils le paieront cher : à de très rares exceptions près, cette génération ne survivra pas aux répressions staliniennes et à la deuxième guerre mondiale. D'ailleurs, un volet de ces mêmes répressions leur est consacré, avec des accusations ad hoc, dont la plus spécifique est celle d'être des espions pour le compte d'une Finlande et d'une Estonie impérialistes. Toute référence à la parenté finno-ougrienne est criminalisée pendant les années 1933-1940 (Toulouze 2006). Toute tentative de développer les langues vernaculaires autrement que par l'emprunt massif au russe est taxée d'entreprise nationaliste et contrerévolutionnaire. Mais dans l'inconscient collectif, quelque chose reste de la période héroïque où les hommes de lettre sont porteurs des destinées du peuple. Les écrivains ne cesseront pas de se sentir responsables.

Dans les régions boréales, la naissance d'une couche intellectuelle est voulue par le pouvoir soviétique lui-même, et ce trait historique la marquera pour plusieurs décennies. Afin de pouvoir efficacement contrôler une région stratégique, celle du passage maritime du Nord, d'une part, et de permettre un dialogue entre les communautés et les autorités d'autre part, il manquait un groupe de médiateurs autochtones, mais capables de faire circuler l'information entre les deux pôles, capables de comprendre (et de soutenir) le projet soviétique, et de faire entendre (pense-t-on ${ }^{5}$ au début) la voix des communautés. C'est ainsi que naissent les intellectuels du Nord en tant que catégorie, recrutés par le pouvoir soviétique et travaillant exclusivement dans les intérêts de celui-ci, convaincus de faire œuvre de construction d'une vie meilleure. Pleins de gratitude, ils ne mettront pas en cause le pouvoir qui leur garantit un statut, une activité, qui leur a ouvert un monde passionnant; ils le servent fidèlement. Il faudra que le vernis commence à craquer, dans les années 1970, pour que les intellectuels s'affranchissent de cette tutelle assumée.

\section{La naissance d'un mouvement finno-ougrien des écrivains}

Les mouvements sociaux de coordination des Finno-Ougriens, surtout des intellectuels, ne sont pas chose entièrement nouvelle: le "mouvement finno-ougrien » a pris naissance dans les années 1920 (Hõimusidemed 1997) dans les nouveaux États indépendants, la Finlande, l'Estonie et la Hongrie, avec le soutien actif des plus hautes instances des trois pays ${ }^{6}$. Mais dans ce mouvement, les écrivains ne jouaient pas de rôle particulier, même si les unions des écrivains faisaient partie des organisations responsables dans chacun des pays. Il faudra attendre pour cela le renouveau des années 1980 en Union soviétique

19 Cette période a connu, en URSS, des transformations structurelles qui traversent la société tout entière. Sans doute, pour les peuples finno-ougriens, c'est le volet glasnost' de la perestrojka qui est le plus bouleversant. La remise en cause du mode de fonctionnement économique de la société soviétique, le passage à l'économie de 
marché, n'est pas pour les Finno-Ougriens de Russie un objectif en soi (au contraire : de manière générale, ces communautés, qui sont dans leur immense majorité agraires, sont attachées au mode collectif de production et n'aspirent pas à la liberté du marché - les changements les ont même affectées négativement). Mais la restructuration économique s'accompagne de l'explosion de la liberté de parole, qui, elle, touche très directement les intellectuels. Encore restent-ils prudents. Dans les régions finnoougriennes, les bouches ne s'ouvrent pas tout de suite. Les souvenirs des répressions se sont transmis de génération en génération; même lors de la déstalinisation, dans les années 1960, ce sont les «nationaux » qui ont été le plus réticents à réhabiliter leurs grands hommes condamnés dans les années 1930 (sur l'épopée de la réhabilitation de Kuzebaj Gerd, cf. Kuvšinova 1990, p. 4, Kuznecov 1994, p. 422).

C'est d'abord dans le monde russe que l'on s'empare puissamment des nouvelles possibilités et que le voile pudique qui recouvrait le passé est levé impitoyablement. Dans le monde finno-ougrien, il faudra attendre la fin de la décennie pour que les bouches s'ouvrent. La première occasion institutionnelle de le faire est offerte aux intellectuels finno-ougriens par une décision courageuse, celle de l'écrivain mari Miklaj Rybakov: ministre de la Culture de la république autonome des Maris, qui n'est pas encore officiellement le Mari El, il invite en 1989 les écrivains et hommes de lettres finno-ougriens à se rassembler à Joškar Ola, pour parler de leurs problèmes communs (Toulouze 1991). L'invitation est large : ne sont pas seulement concernés les écrivains proprement dits, mais plus généralement les hommes et femmes de lettres, les spécialistes de littérature, les traducteurs, les journalistes. Et c'est l'une des premières occasions où les Finno-Ougriens hongrois et finlandais sont associés à leurs collègues soviétiques (l'Estonie n'est pas encore officiellement indépendante, même si des vents de liberté y soufflent déjà).

21 Les participants à cette conférence font éclater les mythes construits par un discours soviétique qui n'avait jusqu'alors jamais été remis en cause de manière aussi compétente. D'après ce discours, la question nationale en URSS avait été résolue. Dans le cadre de la marche au communisme, les différences entre les ethnies se réduisaient et l'homo sovieticus était en train d'émerger. Entre-temps, les cultures s'épanouissaient, nationales dans la forme et soviétiques dans le contenu, soutenues par les autorités, qui leur assuraient un financement adéquat. Au cours de leur rencontre, les écrivains finno-ougriens ne parleront que très peu de littérature. Ils s'inscrivent ainsi dans la lignée des premières autonomies des années 1920, quand ils étaient porteurs des destinées de leurs peuples: ils parlent de démographie, d'éducation, d'édition, de tensions interethniques, de fausse égalité, d'oppression masquée, de discrimination linguistique, de russification. Les chiffres varient de région à région mais les phénomènes se retrouvent partout, il n'y a pas de voix discordante. Les statistiques montrent alors une croissance démographique de chaque peuple (avec bien sûr quelques exceptions, mais elles restent marginales), mais avec une réduction spectaculaire des locuteurs des langues vernaculaires. Autrement dit: plus de personnes sont prêtes à se reconnaître dans les ethnies minoritaires, mais beaucoup d'entre elles ont perdu leur langue d'origine et ont été spectaculairement russifiées. Cette russification est en apparence choisie: les parents ne transmettent pas leur langue et leur culture à leurs enfants, notamment en milieu urbain. De plus, à l'école, l'apprentissage des langues vernaculaires, qui ne sont plus présentes qu'en qualité de matières enseignées, devient optionnel. Mais le choix qui est fait la plupart du temps de ne pas surcharger l'enfant avec une matière supplémentaire - est souvent un choix 
de survie, qui s'explique par la pression du corps social: racisme vis-à-vis des nationaux, mépris ethnique, désespérance. Pour leurs enfants, les parents ne prennent pas de risque.

À la tonalité de lamentation qui caractérise l'ensemble des interventions qui dénoncent pour la première fois des situations vécues par des centaines de milliers de personnes, s'oppose la combativité des Estoniens qui sont en plein combat pour l'indépendance, et ce avec une vitalité incomparable de la langue et du sens d'appartenance à une communauté qui n'a rien de soviétique : Arvo Valton pose une série de revendications ambitieuses, qui font sentir aux minorités en territoire russe que tout, peut-être, n'est pas perdu. Entre autres il demande l'enseignement en langue nationale depuis l'école maternelle jusqu'à l'université. Il donne ainsi une leçon de dignité à tant de FinnoOugriens habitués à être méprisés.

\section{Le développement du mouvement des écrivains finno- ougriens}

À la fin de la rencontre de Joškar Ola, les écrivains promettent de se retrouver. Ils découvrent non seulement le bonheur de pouvoir parler librement de ce qui leur fait mal, mais aussi l'euphorie d'être entre amis, de se serrer les coudes. D'ailleurs, les années qui suivent seront difficiles pour eux : ils verront l'actualité échapper de plus en plus aux intellectuels, ce ne sont pas eux qui seront les protagonistes des changements radicaux qui secouent l'URSS et qui en provoquent l'écroulement. Pire, alors que sous le régime soviétique ils avaient une position sociale privilégiée, l'économie de marché les ignore.

En même temps, le relais est pris par les Finlandais. En effet, ils viennent tout juste de créer, en 1990, une institution ad hoc: l'association Castrén 7 , a pour mission de gérer les relations avec les Finno-ougriens de Russie. Ces relations s'intensifient ; il est désormais plus facile de voyager. En 1990, par exemple, il y a un lecteur de mari à l'université d'Helsinki. Il est désormais possible de se rendre dans des régions naguère encore fermées. Ce ne sont en fait pas les écrivains finlandais - qui ont une expérience historique différente dans leur rôle social - mais des universitaires, spécialistes des langues et des cultures finno-ougriennes, qui organisent le deuxième congrès des écrivains finno-ougriens, tenu Espoo, près de la capitale, en août 1991, sous le mot d'ordre « Kieleni on kotimaani » (Ma langue est mon pays). L'Estonie n'est toujours pas indépendante - mais ce n'est plus qu'une question de jours, le putsch à Moscou éclate deux jours après la fin des travaux. Nombreux sont les intellectuels de peuples finnoougriens de Russie présents ; il y a même des Nénètses qui, pour la première fois, sont associés à ces initiatives. Le président Mauno Koivisto accueille tous les participants avec une réception, portant ainsi l'initiative au plus haut niveau politique. Les langues y sont vraiment à l'honneur : les intervenants parlent leur langue, et une traduction simultanée est assurée dans les principales langues internationales (finnois bien sûr, hongrois, anglais, russe). Du point de vue du contenu, la langue y est présentée comme l'élément central de l'identité nationale, ce qui correspond à l'expérience historique de la plupart des participants. C'est une somptueuse conférence: en Finlande, on sait organiser; les participants sont hébergés dans des résidences universitaires modernes et fonctionnelles, beaucoup d'entre eux découvrent un certain monde occidental qui leur est accueillant. 

de coordination des écrivains finno-ougriens qui puisse fonctionner en permanence. Mais on en était resté à l'expression d'un souhait général. Les Estoniens veulent désormais aller plus loin et créer véritablement une association internationale. En fait, il faut souligner, déjà à ce moment-là, le rôle personnel d'Arvo Valton, écrivain de renom, connu pour son hostilité au régime soviétique et passionné de solidarité finnoougrienne. En effet, la plupart des intellectuels estoniens regardent vers l'Ouest, ils ne sont guère intéressés par des liens qui conduisent surtout vers la Russie profonde - la Finlande leur est de toute manière ouverte. Mais A. Valton est vice-président de l'Union et il arrive, avec le prestige de sa personne, à préserver cette dimension finnoougrienne.

L'actualité va vite : les participants estoniens n'ont pas encore quitté Helsinki que l'on apprend que les tanks défilent dans Tallinn. Les communications téléphoniques et le trafic maritime ne sont pourtant pas coupés. Quelques jours plus tard, l'Islande reconnaît l'indépendance de l'Estonie, suivie par beaucoup d'autres pays. La Russie suit le 6 septembre. El'tsin prend une avance décisive dans sa confrontation avec Gorbačëv, la Russie contre l'Union soviétique. L'Union soviétique n'a plus que quelques mois à vivre.

le monde intellectuel finno-ougrien de Russie. L'initiative de Rybakov a éveillé l'appétit de rencontre. Quelques intellectuels se mettent d'accord pour constituer une Union des écrivains finno-ougriens de Russie : elle est présidée par le Carélien Robert Vinonen (un universitaire poète), et a pour vice-présidents Semën Samsonov (un Oudmourte) et Juvan Šestalov (le «monstre sacré » mansi). Les Unions des écrivains jouent encore un rôle important presque partout en Europe de l'Est: la constitution d'une Union «transethnique» est un exemple original qui mérite d'être noté, et qui est bien représentatif de son époque.

\section{Vers la constitution d'une association}

s'écrouler. Les structures sont partout en crise, une crise alimentaire sévit partout, même dans l'Estonie nouvellement indépendante. En Finlande aussi, c'est la crise - lama - et la population est en état de choc. En Hongrie, ce n'est pas mieux. C'est donc dans un contexte particulièrement difficile que les Estoniens prennent l'initiative de convoquer non point une assemblée générale, mais une réunion de travail des Unions finnoougriennes des écrivains fin janvier à Tallinn.

Participent à cette réunion des représentants des Unions finno-ougriennes d'écrivains. De Finlande, Jarkko Laine et Leena Laulajainen, cette dernière ayant écrit des livres pour enfants et ayant découvert, avec un enthousiasme de néophyte, le monde mari ; l'Union hongroise avait délégué son secrétaire général, Sándor Koczkás, et le grand spécialiste des littératures finno-ougriennes, Péter Domokos; les trois responsables de l'Union de Russie étaient présents. Demeurant en Estonie à l'époque et étant intéressée à ces questions, j'ai été associée à ces travaux et, désormais, je serai impliquée dans l'ensemble des initiatives du mouvement. Mon expérience et mes souvenirs me serviront de sources dans la suite de cette étude. 
30 La rencontre a fait émerger un obstacle inattendu à la concrétisation des projets d'association. En effet, alors qu'un bel accord se dessinait, les délégués finlandais ont mis leur veto à la création d'une association internationale. Manifestement, ils avaient peur d'être piégés dans un système où ils ne seraient pas majoritaires et où ils risquaient de devoir avaliser des décisions qui ne les concerneraient pas. Et puis la Finlande était en crise ; malgré tout, elle avait, parmi tous les présents, la puissance économique la plus solide, et sans doute l'Union des écrivains craignait-elle de devoir se retrouver dans une position de vache à lait. Toujours est-il que les positions et les comportements des délégués finlandais ont permis de mesurer l'écart entre ceux qui venaient $\mathrm{du}$ monde socialiste (soviétique ou non) et les Finno-ougriens les plus « occidentaux ».

31 L'aspiration de la majorité des personnes concernées ne pouvait cependant pas être arrêtée par le désir de l'un des membres potentiels de rester extérieur à l'entreprise. Le processus de création d'une association s'est poursuivi, sans les Finlandais mais avec une double locomotive hungaro-estonienne. L'union hongroise des écrivains s'était en effet engagée à Helsinki à organiser la troisième rencontre en 1993, à Eger. La préparation de la conférence d'Eger a conduit à mettre en place le cadre juridique pour la création de l'association et a vu plusieurs rencontres courant 1993 entre A. Valton, S. Koczkás, p. Domokos et moi, pour élaborer des textes et les traduire dans les diverses langues concernées.

32 Comme les rencontres précédentes, celle d'Eger a été organisée à un très haut niveau. Le président hongrois Árpád Göncz l'a honorée de sa présence. Il venait d'ailleurs de faire en Russie une tournée triomphale, une première historique : atterri à Moscou, il avait fait le tour des régions finno-ougriennes, rencontrant non seulement les autorités de l'État mais aussi les responsables des mouvements nationaux. C'était donc là l'occasion de retrouvailles avec des intellectuels qu'il avait rencontrés sur place quelques mois auparavant. Inutile de dire qu'une telle initiative de la part du président de la Hongrie avait été extrêmement populaire dans les régions finno-ougriennes.

Du point de vue du contenu des interventions, il faut noter très peu de changements par rapport aux rencontres précédentes: une sorte de nouveau discours canonique semblait se mettre en place, relevant de la lamentation. Mais la proposition concrète des organisateurs hongrois de mettre en place une association a permis de tourner les travaux vers des questions plus constructives. Les Finlandais quittèrent la rencontre. Mais les autres travaillèrent avec enthousiasme à l'adoption des statuts et de tous les textes nécessaires à enregistrer l'association en Hongrie. Les séances furent d'ailleurs suivies par un fonctionnaire de l'ambassade de Russie, qui ne se priva pas de travailler avec insistance auprès des minorités de Russie, en essayant de les tourner contre les Estoniens (accusés d'avoir provoqué l'écroulement de l'URSS) et contre la création de l'association. C'est un signe des temps que ces tentatives d'intimidation aient échoué.

34 La question de la direction de l'association souleva bien des débats. En effet, Péter Domokos, qui a été le premier à poser la question des littératures finnoougriennes dans le monde universitaire et à faire connaître les auteurs des diverses régions en dehors de leurs aire d'influence, était de loin la personnalité la plus connue et la plus influente de toutes. Domokos lui-même avait proposé que fût élu Arvo Valton. Mais celui-ci n'était pas encore suffisamment connu dans les régions, et sans doute la pression de l'ambassade de Russie a-t-elle également joué, peu ou prou: les représentants des littératures finno-ougriennes élurent Domokos à l'unanimité. 

pratiquement tous les deux ans. Le premier congrès de la toute nouvelle association devait avoir lieu à Saransk, en Mordovie, en 1995. Mais, malgré les promesses des écrivains mordves à Eger, la Mordovie n'était pas prête. C'est donc l'Estonie qui a pris le relais.

\section{Vers la stabilisation}

Pour organiser un Congrès alors même que le désistement mordve était arrivé courant 1995 , il fallait un peu plus de temps que quelques mois. Pour la première fois dans son histoire, le mouvement des écrivains finno-ougriens fit une pause de trois ans et non de deux, entre 1993 et 1996, entre Eger et Lohusalu en Estonie.

Si le congrès de Lohusalu a été organisé nominativement par l'Union des écrivains d'Estonie, en réalité il l'a été par A. Valton seul, ou plutôt assisté d'une équipe qu'il avait recrutée lui-même. En fait, la représentation estonienne y a été faible, les regards des écrivains estoniens étant, à ce moment-là, de plus en plus tournés vers l'Ouest - la Scandinavie, l'Allemagne principalement. Mais l'État estonien s'est engagé sérieusement dans l'organisation de ce congrès, qui donc n'a pas eu à souffrir du désintérêt progressif de l'Union des écrivains. Comme dans les cas précédents, les participants ont été accueillis par le Président de la République dans les jardins du Palais de Kadriorg, Lennart Meri étant particulièrement intéressé, à titre personnel, par la promotion de la parenté finno-ougrienne. Il connaissait d'ailleurs personnellement bien des participants.

Figure 1.

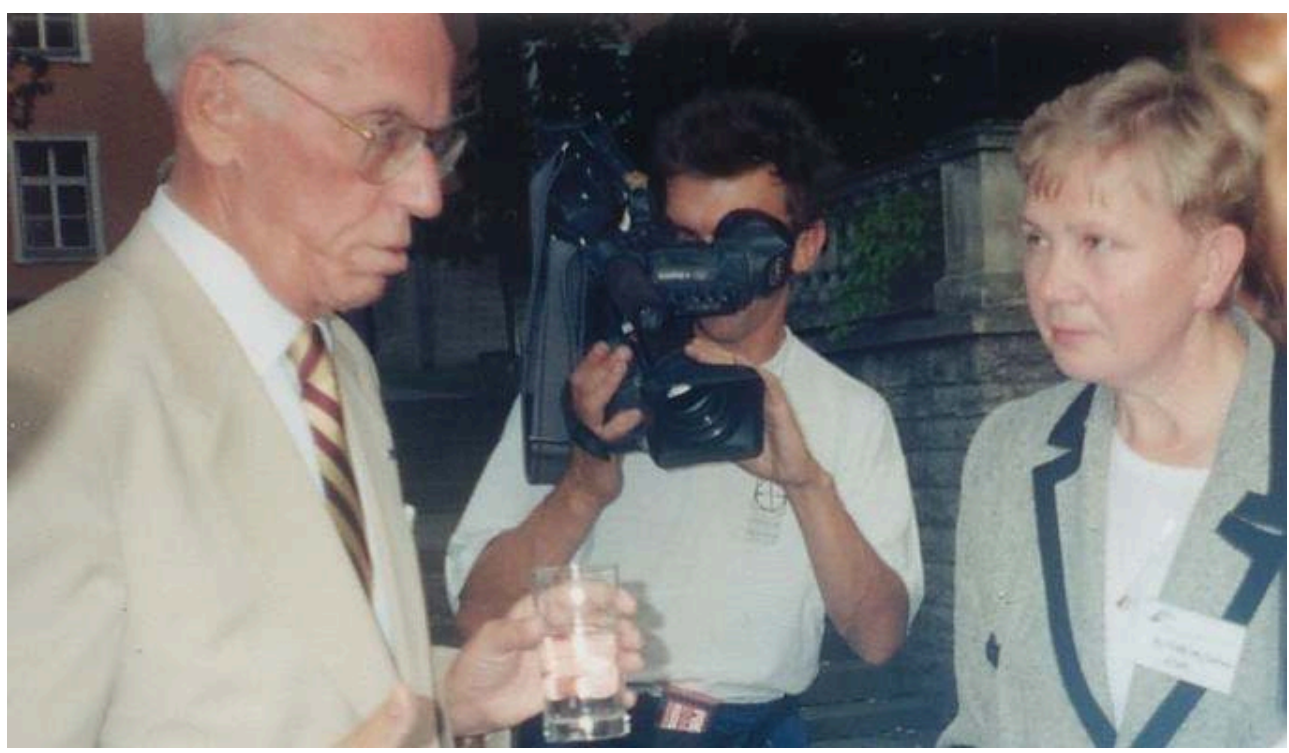

Lennart Meri, président de la République d'Estonie, s'entretient avec Galina Butyreva, poétesse komie, vice-présidente de la Douma komie.

En comparant le contenu de cette rencontre avec celles qui l'avaient précédée, Lohusalu marque un véritable tournant. Plus qu'un grand rassemblement solennel, elle s'est présentée comme une réunion de travail, moins formelle et plus efficace, plus littéraire et moins politique, avec des sections spécifiques par genre. En plus, le ton 
donné par les organisateurs tendait à éviter les lamentations et à se concentrer sur le travail qu'il était possible et souhaitable d'accomplir en vue d'atteindre des objectifs communs. Le mot d'ordre du congrès était "Sillad " (ponts), et l'idée était de mettre l'accent sur les traductions mutuelles des littératures finno-ougriennes. De mieux se connaître, se désenclaver, dialoguer. En fait, le Congrès d'Eger avait déjà produit une anthologie de poésie finno-ougrienne traduite en différentes langues (Megyek... 1993). Tout le monde pouvait contribuer à cet objectif, et la démarche projet par projet ne pouvait que faciliter l'obtention de subventions de sources diverses. C'est aussi à Lohusalu que paraîtra le premier numéro d'INF, bulletin du Congrès avec des informations sur l'état actuel des littératures dans chaque région. Un numéro sera publié lors de chacun des congrès suivants.

Ce congrès marque également le retour des Finlandais : sans engagements solennels, des représentants de l'Association Castrén y étaient néanmoins présents (Johanna Laakso, Jouni Tossavainen, Anita Konkka) et actifs. On constatait un désengagement de l'Union des écrivains en tant que telle, mais les écrivains motivés par les contacts avec les Finno-Ougriens étaient activement là.

40 À Lohusalu, finalement, Domokos s'est retiré comme il pensait le faire trois ans plus tôt. A. Valton était désormais connu et apprécié. Son élection n'a posé aucun problème et il a pris alors pour quatorze ans les rênes du mouvement.

Depuis Lohusalu, tous les congrès se sont concentrés sur la question des traductions mutuelles :

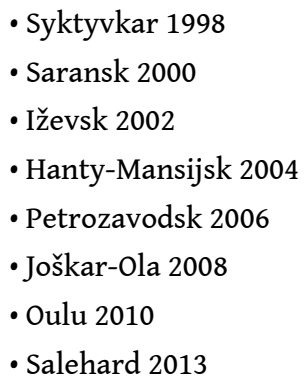

Il faut noter que chaque congrès a revêtu une importance toute particulière pour la région organisatrice. Il a permis aux écrivains de la nationalité éponyme d'acquérir de la visibilité, d'obtenir des financements, de publier des livres. Le fait qu'une partie de participants venait de l'étranger a permis aussi de faire de ces rencontres une vitrine. En même temps, elles ont bien reflété la situation de la culture autochtone dans les différentes régions. De manière générale, les autorités des Républiques et des régions se sont pleinement engagées dans l'organisation de ces journées.

Certaines rencontres ont été plus solennelles que d'autres : à Saransk et à Iževsk, elles ont été organisées dans de grandes salles de conférences du centre-ville, avec un protocole élaboré. D'autres ont été plus simples. Au Mari El, qui avait eu une certaine heure de triste célébrité au niveau européen en raison des accusations qui lui avaient été adressées de violer les droits des Maris, l'organisation a été impeccable : le ministre de la Culture lui-même, un Mari, a participé activement aux travaux et aux discussions, avec intelligence et modestie.

44 L'organisation d'excursions pour les participants a bien révélé la part des valeurs autochtones dans la vision par les autorités de ce que leur région a à offrir aux visiteurs : si à Syktyvkar les écrivains finno-ougriens ont été emmenés au village natal 
du premier écrivain komi Ivan Kuratov, ou à Joškar Ola, au village natal de Sergej Čavajn après avoir visité l'école de Morko, où la langue marie est très présente, à Saransk la visite a porté sur un monastère orthodoxe (montrant la perception de l'orthodoxie comme parfaitement intégrée à l'identité mordve) et à Iževsk, les écrivains ont été conduits non pas au musée Gerd dans l'école où celui-ci a enseigné, mais au musée Tchaïkovski à Votkinsk...

Figure 2.

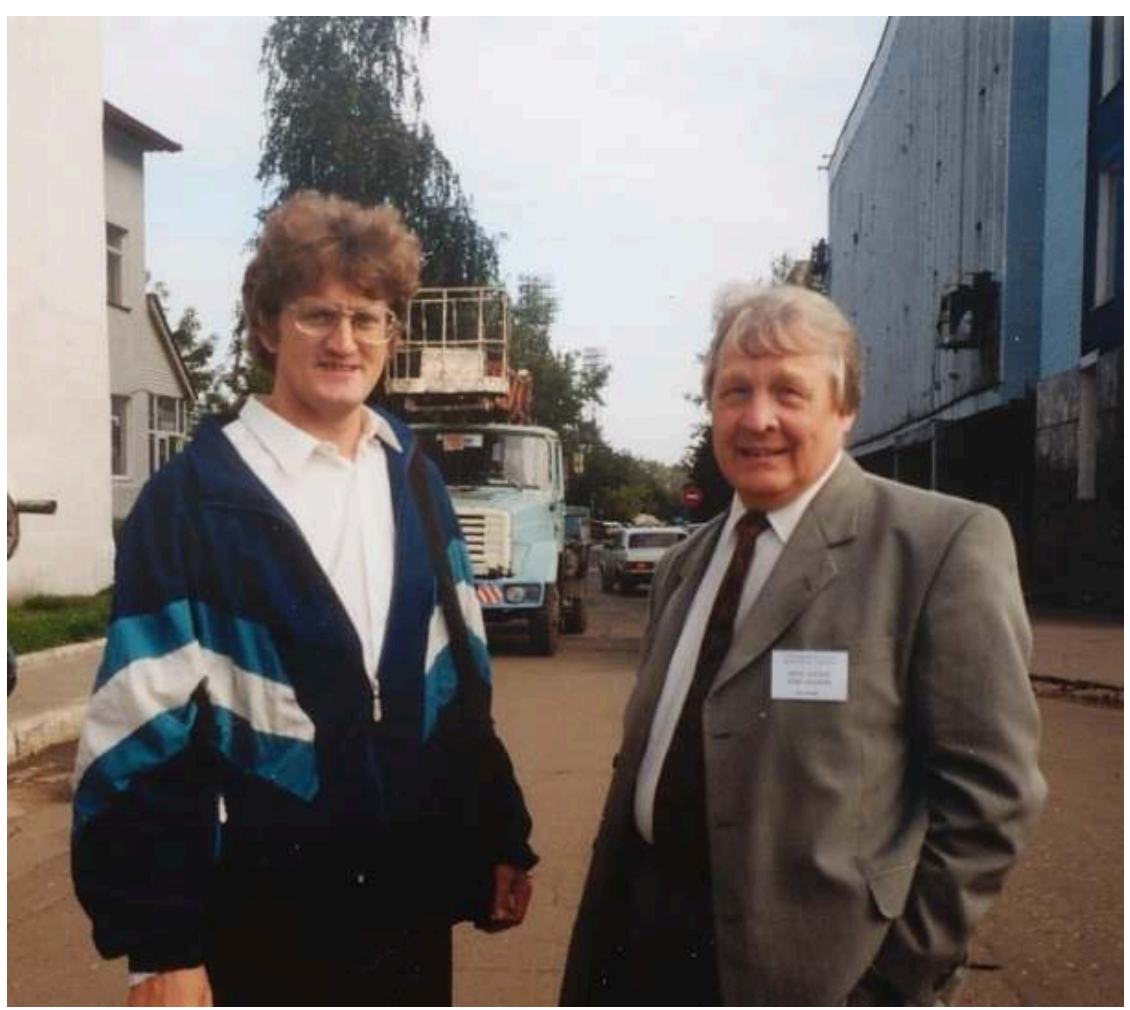

Arvo Valton, le président de l'Association des littératures finno-ougriennes, avec le poète et chercheur oudmourte Viktor Šibanov à Saransk en 2000. 
Figure 3 .

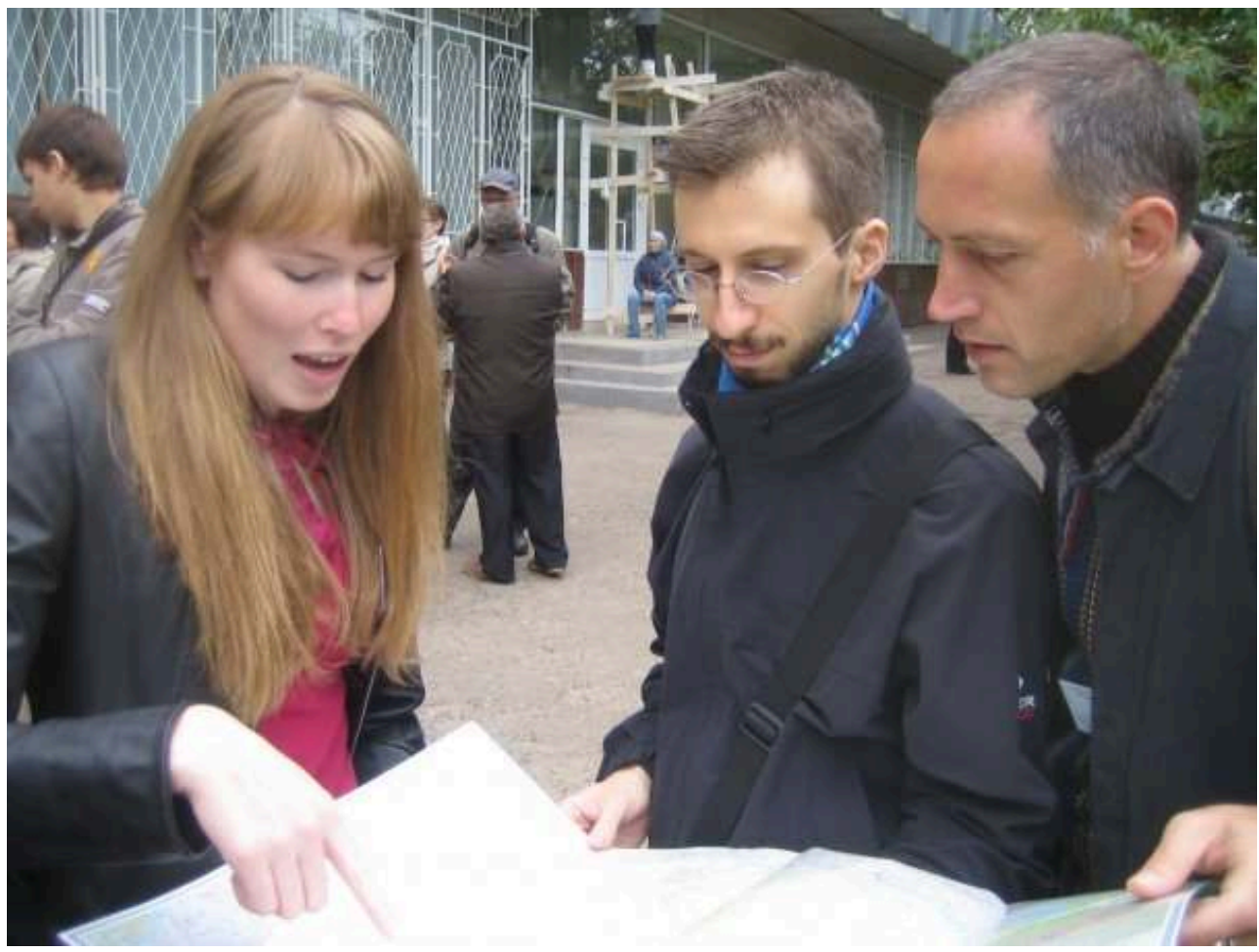

Sébastien Cagnoli et Vincent Lorenzini avec la guide marie, Oksana, à Joškar Ola en 2008.

Figure 4 .

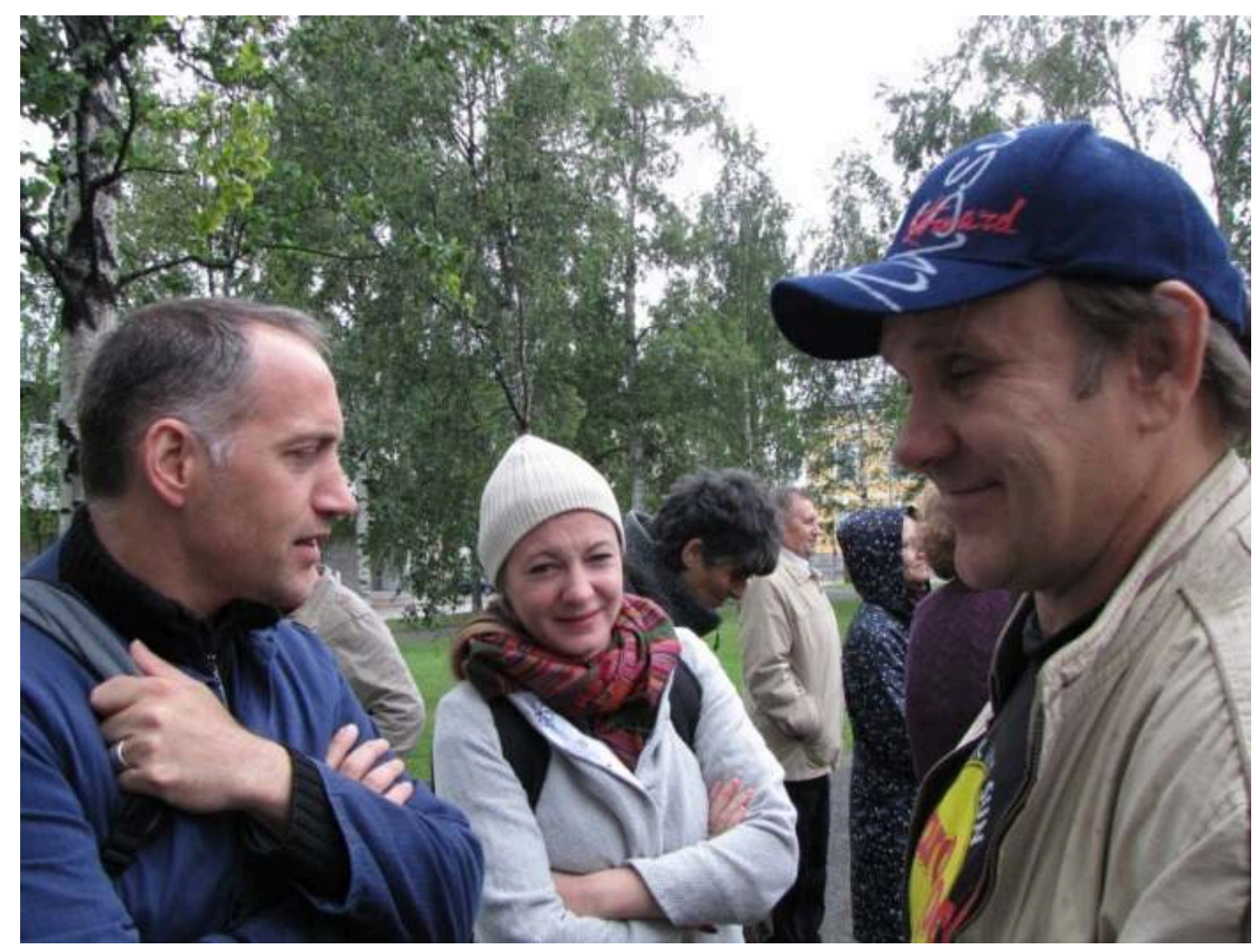

Vincent Lorenzini et Marie Casen en compagnie du poète vepse Nikolaj Abramov à Oulu en 2010. 
Le principal acquis de l'association a certainement été la multiplication des traductions mutuelles. Il va de soi que l'association n'a fait que servir de cadre; elle a permis de donner l'impulsion: l'essentiel du travail est dû à l'engagement de personnalités travaillant souvent seules ou presque. En Estonie, le président Arvo Valton s'est engagé à fond et avec enthousiasme dans le travail de traduction et de publication d'écrivains finno-ougriens jeunes et moins jeunes. Il a créé auprès d'un éditeur militant comme lui deux collections, l'une de recueils de poèmes d'auteurs finno-ougriens traduits en estonien, l'autre également de poésie, mais avec des poèmes en langue originale avec traductions dans trois langues : estonien, russe et anglais. En russe et en anglais les traductions sont faites mot à mot, afin de permettre l'accès à un public international, éventuellement de traducteurs. Cette collection a d'ailleurs inspiré le Hongrois János Pusztay, qui a lancé à Szombathely une version de cette même collection avec les mêmes originaux traduits en hongrois, allemand et français. En Estonie, ces séries comportent déjà quelques dizaines d'ouvrages chacune. De plus A.Valton a publié plusieurs recueils spécifiques: trois recueils bilingues de classiques de la poésie (original/estonien), et quatre recueils bilingues de poésie féminine (quatre poétesses oudmourtes, quatre poétesses maries, quatre poétesses mordves, quatre poétesses komies). Il a aussi traduit une volumineuse anthologie de poésie oudmourte et un recueil de poèmes de Kuzebaj Gerd. Récemment, il s'est tourné vers la prose, et des anthologies de prose de tous les peuples finno-ougriens de la Volga ont paru en estonien. Quelques autres traducteurs estoniens ont cependant relevé le défi et publié, chez des éditeurs renommés, de la prose finno-ougrienne : Eremej Ajpin a été traduit du russe par Peeter Volkonski ${ }^{8}$ et Juri Vella ${ }^{9}$ par Art Leete.

En Hongrie, c'est Katalin Nagy qui porte le flambeau des traductions, avec plusieurs recueils d'auteurs sibériens (Ajpin, Vagatova ${ }^{10}$, Moldanova ${ }^{11}$, Dinislamova ${ }^{12}$ ), et plus récemment avec un recueil bilingue du poète vepse Nikolaj Abramov ${ }^{13}$. Mais János Pusztay a lui aussi édité un recueil de poèmes de Juri Vella, traduit par luimême ${ }^{14}$.

47 L'expérience estonienne a fait boule de neige. L'intérêt pour les traductions mutuelles a augmenté partout et cet intérêt a été alimenté entre autres par un prix littéraire décerné par l'association estonienne Fenno-ugria qui comporte une catégorie traduction. Les recherches de Nadežda Pčelovodova ${ }^{15}$ en portent témoignage. La principale évolution que l'on note dans les dernières années est la pénétration de la prose. C'est clair avec les traductions d'A. Valton en estonien, mais aussi avec la traduction en finnois du roman classique mari, Elnet (1937) de S. Čavajn ${ }^{16}$.

\section{Les difficultés du mouvement des écrivains finno- ougriens}

Ce mouvement est cependant confronté à plusieurs difficultés, certaines d'entre elles structurelles, d'autres plus conjoncturelles.

\section{L'absence des Sames}

Dès le début, le mouvement présente une faiblesse sensible, qui tient à l'éloignement et à l'absence d'une composante importante du monde finno-ougrien, celle des Sames. Il 
existe une littérature same ${ }^{17}$ et les Sames des différents pays ont rencontré des succès remarquables, ces dernières décennies, sur le plan politique. Les Sames ont pourtant jusqu'ici ignoré ce mouvement. Deux écrivains étaient présents à Espoo en 1991 : Kirsti Paltto et Rauna Paadar-Leivo, toutes deux de Finlande. Si Kristi Paltto s'est rendue en Estonie en 1996, il faudra attendre Oulu, en 2010, pour qu'un Same, Niillas Holmberg, fasse entendre sa voix, et ce avec un impact considérable, puisqu'il a été le vainqueur du slam poétique organisé à cette occasion.

Comment expliquer ce boycott? Sans doute, les Sames trouvent-ils une caisse de résonance plus efficace dans d'autres cadres, comme le cadre circumpolaire. C'est d'ailleurs là une raison qui explique aussi la tiédeur relative de l'ensemble des peuples du Nord, y compris ceux de la Russie, qui ont certes été présents, mais plutôt en pointillé. Mais est-ce là tout? On a le sentiment que pour les Sames, le lien finnoougrien reste étranger et artificiel, alors que leur tout premier cadre de référence est scandinave, nordique, arctique, circumpolaire. Cette explication ne me convainc pas entièrement mais je n'en ai pas de meilleure.

Figure 5.

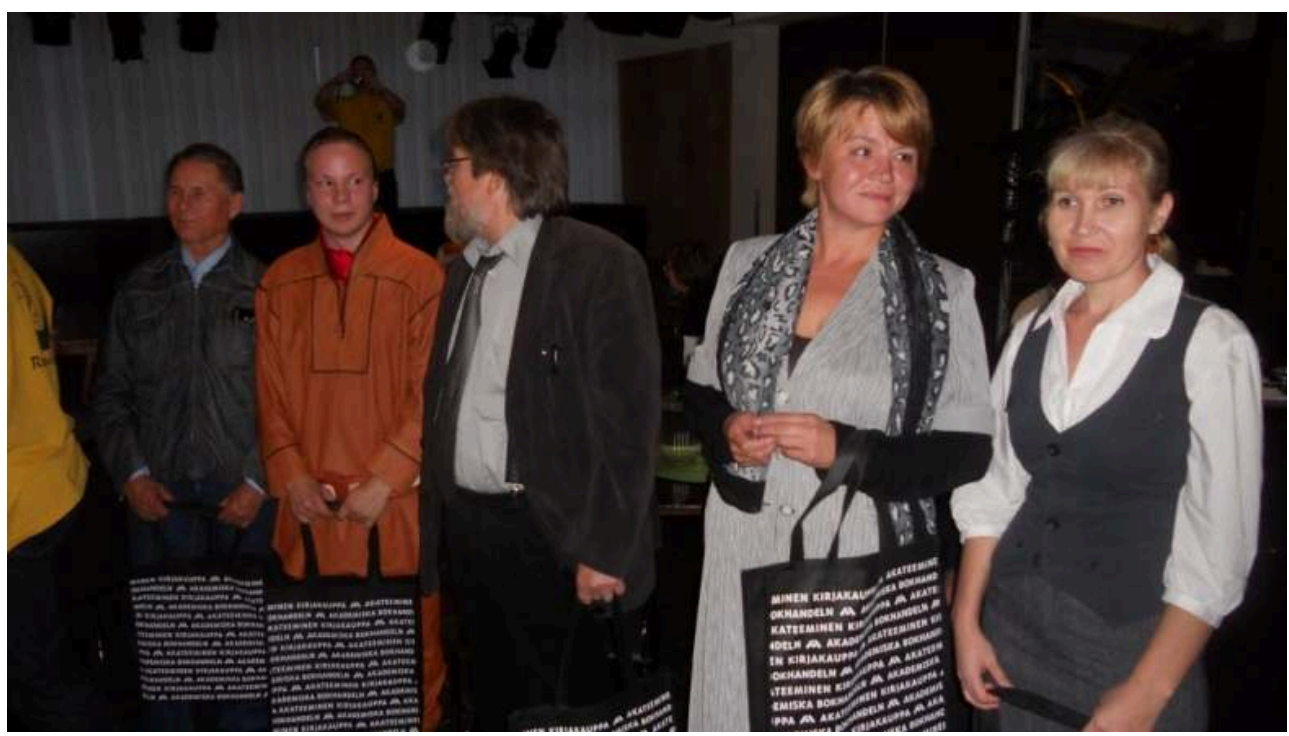

Les lauréats du slam poétique finno-ougrien en 2010 à Oulu :(de gauche à droite) Vasilij Vanjušev (poète et chercheur oudmourte), Niillas Homberg (poète et musicien same), l'animateur du slam, Nina Obrezkova (poétesse komie) et Svetlana Arhipova (poétesse marie).

\section{Une association à structuration lâche}

Qui donc sont les membres de cette association? Plusieurs fois la question a été débattue dans les organes de direction. Ne faudrait-il pas délimiter la portée de l'association et fixer une fois pour toutes la qualité de membre? La réponse a en général été de laisser les choses en l'état et de profiter des avantages que donnent des effectifs indéterminés. En effet, quelle est la situation actuelle?

Les unions finno-ougriennes d'écrivains sont considérées membres ex officio. Par leur intermédiaire, chacun de leurs membres est membre de l'association internationale. Voici déjà un point de départ qui n'est pas sans poser des problèmes. Tout d'abord, comme l'expérience finlandaise nous l'a montré, les Unions des écrivains n'ont pas 
toutes la même fonction ni la même portée partout. Dans certains pays, comme en Hongrie, il y a même aujourd'hui plusieurs Unions des écrivains. Et puis dans les différentes régions de Russie, elles ont une politique plus ou moins fédératrice ou, à l'inverse, plus ou moins sélective : suivant l'endroit, beaucoup d'écrivains, aujourd'hui, ne sont plus membres de l'Union, qu'ils n'y soient pas admis ou qu'ils n'aient aucune motivation pour y adhérer. En Oudmourtie, par exemple, un PEN club s'est créé en alternative, qui a également rejoint l'Association internationale. La direction de l'association ne veut pas exclure des écrivains qui, pour des raisons diverses, ont choisi de ne pas être membres de leurs associations locales. De plus, il y a également des personnes extérieures qui peuvent jouer un rôle dans la vie de l'Association : je fais partie depuis 1996 de sa direction, ce qui laisse supposer que j'en suis considérée comme membre à titre individuel. Donc, il y a des membres collectifs et des membres individuels.

Les structures de direction sont également un signe de cette flexibilité. Le conseil de direction a un président qu'il élit, d'après les statuts, en son sein. Après Péter Domokos, Arvo Valton a été président pendant quatorze ans et a été remplacé au Congrès d'Oulu par le Finnois Kari Sallamaa ${ }^{18}$. Le président est assisté de deux vice-présidents tournants, qui sont les représentant de la région ayant organisé le dernier congrès et de celle qui organise le congrès en cours. Le conseil de direction se compose sur le principe d'un représentant par ethnie. En même temps, dans beaucoup de cas, des problèmes se posent : certaines ethnies, comme les Nenetses, sont représentées dans différentes entités territoriales, plus précisément dans trois arrondissements autonomes. Or les unités territoriales sont réellement des entités séparées, souvent sans contact entre elles. Donc, il serait opportun d'avoir à la direction un représentant non seulement par ethnie, mais aussi par unité territoriale... Souvent, ces problèmes se résolvent facilement de manière empirique. Pendant les premières années du fonctionnement de l'Association, deux Nenetses participaient au travail de l'association : Elena Susoj, du Jamal, et Prokopij Javtysyj, du District autonome nenetse. Une place a été donnée aux deux. Aujourd'hui, Javtysij n'est plus là, Susoj s'est retirée et c'est Nina Jadne qui, seule, venue de Nadym, dans le Jamal, représente les Nenetses de la toundra. Ensuite se pose la question, par exemple chez les Khantys, des groupes ethniques qui ont des langues entre lesquelles l'intercompréhension est difficile. Maria Vagatova a suivi les travaux dès le début. Mais dès lors qu'Eremej Ajpin a été libéré de la plupart de ses responsabilités politiques de haut niveau, il a semblé pertinent de l'associer aux travaux de l'association et de sa direction. Cela fait déjà deux représentants khantys, une du Nord, l'autre de l'Est. En fait, depuis que J. Šestalov et A. Tarhanov se sont éloignés de l'association, il n'y a plus de Mansis représentés, mais le district autonome des Khantys et des Mansis est habité également par des Nenetses des forêts: de son vivant, Jurij Vella a toujours été sans aucune hésitation invité à participer aux réunions de la direction.

Nous avons donc là une entité extrêmement fluctuante, qui ne fonctionne pas selon des principes dits démocratiques, parce qu'il n'existe pas, de manière générale, de communauté bien définie derrière chacun des créateurs présents. On est représentant de soi et on porte l'expérience collective de sa communauté, telle qu'elle est perçue par cette personnalité, et sans contrôle par une " base » éventuelle. Si l'association essayait de fixer les choses, cela serait indiscutablement plus clair, mais aussi plus formel : on aboutirait à une surreprésentation des communautés institutionnellement installées, alors que souvent l'initiative et la vitalité, que l'association entend soutenir, sont 
ailleurs. Jusqu'ici, malgré des discussions répétées sur ce thème, le choix a été fait de continuer de manière empirique et de procéder par cooptation, de manière à avoir dans le conseil de direction des personnes motivées et bénéficiant d'autorité dans leurs régions, voire internationalement.

\section{La question du président}

Il y a en russe deux mots pour "président », un peu comme en anglais «chairman » et «president». La direction, composée en majorité de «Russiens», citoyens de la Fédération de Russie, a insisté pour avoir le mot étranger "prezident ", qui est plus impressionnant. Le premier président a été hongrois, le deuxième estonien, le troisième est finlandais. À quand un président issu de Russie ? La question est délicate. Deux facteurs de prudence, l'un venu de l'extérieur et l'autre de l'intérieur de la Russie, ont fait en sorte que jusqu'à ce jour la direction s'est abstenue. Il est vrai qu'un tel poste s'accompagne d'un prestige certain et que l'autorité du président permet de lever pas mal d'obstacles. En même temps, jusqu'à aujourd'hui, cette autorité allait de pair, en Russie, avec le fait qu'il s'est toujours agi d'un étranger. Or être étranger en Russie confère un statut tout particulier. Est-ce qu'un président lui-même " russien » pourrait être aussi respecté - beaucoup en doutent. Le deuxième facteur est plus négatif. Beaucoup doutent de ce qu'un président originaire de Russie puisse avoir la liberté et la marge de manœuvre qui existent actuellement. La crainte de voir l'organisation être manipulée de l'extérieur, y compris politiquement, par des autorités peu respectueuses de l'intérêt des Finno-Ougriens, existe, et il serait vain de le nier. Ces craintes sont-elles justifiées? Il est difficile de rien dire de sûr, mais l'expérience de l'histoire rend évidemment prudentes, peut-être même à l'excès, les communautés vivant sur ce territoire et ayant connu la pratique de la répression. 
Figure 6.

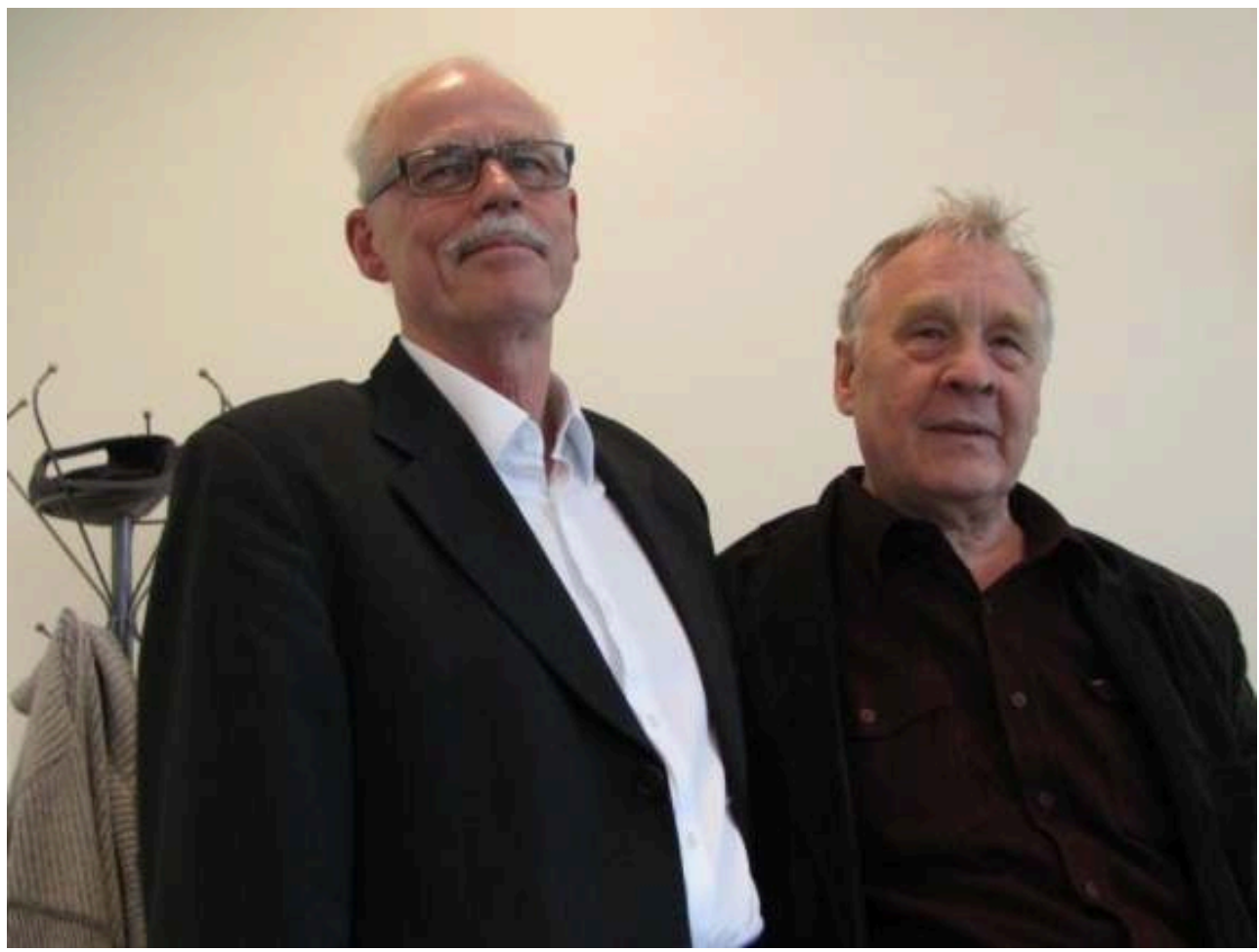

L'ancien président, Arvo Valton (à droite), et le nouveau, Kari Sallamaa, à Oulu en 2010.

\section{Une continuité menacée}

Malgré la motivation des écrivains engagés, le principe de rotation fait ressortir les différences entre les régions et la capacité ou l'incapacité des uns et des autres d'organiser, une fois tous les deux ans, un congrès. En 1993, l'Union des écrivains de Mordovie avait manifesté son désir d'organiser le Congrès en 1995, par la voix de Valentina Mišanina. Puis elle s'était retirée. L'intervalle de deux ans avait été porté à trois pour permettre à un nouvel organisateur, l'Estonie, de se mettre sur les rangs. Nous sommes confrontés au moment où cet article est écrit, à une situation analogue : la Hongrie vient de se retirer, alors qu'elle était censée organiser le Congrès de 2012. Ce retrait est causé par deux circonstances concomitantes : d'une part en 2012, c'est la Hongrie qui organise le Congrès des peuples finno-ougriens, et cela semble être un poids budgétaire qui écarte tous les autres projets finno-ougriens; d'autre part, le gouvernement hongrois ne regarde pas avec sympathie la parenté finno-ougrienne, ce qui explique la période de creux que traversent les organisations qui s'y sont consacrées. Mais ce retrait est un coup sérieux à l'association, car il ne s'agit pas d'une petite région marginale, mais l'un des piliers. L'organisera-t-elle en 2013 ? Une solution alternative a été envisagée, qui est d'organiser le Congrès dans le Jamal ${ }^{19}$.

\section{Conclusion : un bilan}

57 À quoi aura servi ce mouvement ? Quel bilan pouvons-nous en tirer ? 
58

Je crois qu'il y a deux dimensions à prendre en compte, dont l'une est mesurable et l'autre non.

L'apport mesurable est le nombre de traductions mutuelles qui ont été publiées ces dernières années, le nombre d'anthologies, certaines ayant vu le jour à l'occasion des congrès. Seul un petit nombre de ces publications auraient existé sans l'impact des congrès des écrivains : ceux-ci ont donné aux écrivains l'occasion de connaître les œuvres et les auteurs d'autres cultures, l'envie de travailler ensemble, et ont montré que c'était possible. Des étudiants venus de diverses régions de Russie et étudiant à Helsinki $^{20}$, Tartu ${ }^{21}$, Tallinn ${ }^{22}$ ou Budapest ${ }^{23}$ ont appris les langues de ces pays : ils ont aidé à traduire des œuvres de leurs littératures dans leur pays d'accueil, mais ils ont aussi traduit eux-mêmes dans leur langue des œuvres finnoises, estoniennes ou hongroises. Certes, la diffusion de ces œuvres laisse encore à désirer. Mais elles existent, et c'est déjà un point de départ. portée, par nature, reste difficile à évaluer. C'est la dimension psychologique. Il faut d'emblée reconnaître ici ce qu'il est difficile d'avouer ouvertement : les destinataires de ces initiatives sont avant tout les Finno-Ougriens de Russie. Au moment où le mouvement se met en place, ce sont eux qui sont demandeurs de relations internationales : les autres, depuis 1990-91, ont accès au territoire de l'URSS puis de la Russie, et ne se sentent plus, comme auparavant, coupés du reste du monde finnoougrien. Non seulement parce que les frontières sont ouvertes, mais aussi parce que, surtout en Finlande et en Estonie, il est possible de trouver des financements.

Il n'en va pas de même en Russie, et ce pour plusieurs raisons. Il est vrai que les obstacles administratifs au voyage à l'étranger ont été en grande partie levés. Plus précisément, ont été levés les obstacles mis par leur pays à la circulation extérieure. Mais les autres pays restent prudents vis-à-vis des ressortissants de la Russie, connus pour leur désir d'émigration. On peut même dire que l'espace, vu de Russie, se réduit : avant, ils pouvaient se rendre en Estonie sans visa. Désormais, l'Estonie, qui n'a que trop d'immigration léguée par l'ancien régime, distribue ses visas avec parcimonie. De toute manière, obtenir un visa pour l'étranger, quand on est citoyen de la Fédération de Russie, est une épreuve qui demande beaucoup de persévérance. Surtout, à l'obstacle administratif s'ajoute l'obstacle financier. Dans les années 1990, la crise en Russie s'approfondit, les salariés attendaient leur rémunération pendant des mois, les prix des transports intérieurs flambaient ; cela a abouti à un isolement de plus en plus profond : j'ai rencontré en 1997 Fëdor Istomin, président de l'Union des écrivains du district komi-permiak, qui m'a dit que cela faisait plusieurs années qu'il n'avait pas pu quitter le territoire du district. Maintenant la situation s'est stabilisée, mais les transports intérieurs restent chers, sinon prohibitifs, et les financements publics sont rares.

Souvent, les intellectuels des différentes régions ne se retrouvent que lors d'initiatives internationales. Nous, Occidentaux, semblons parfois avoir plus de relations avec chaque communauté qu'ils n'en ont eux-mêmes. Il est incontestable que la pénétration d'Internet et des réseaux sociaux a levé, ces dernières années, le carcan de l'isolement. Mais encore ne sont-ils accessibles qu'à une jeune génération. Beaucoup d'intellectuels cinquantenaires et plus âgés ne se sentent pas à l'aise avec un ordinateur, si tant est qu'ils en trouvent un à leur disposition. Or dans les régions finno-ougriennes, ils continuent toujours à jouer un rôle non négligeable, et beaucoup de militants engagés dans l'association font justement partie de cette classe d'âge. 
soit l'Association Castrén, qui par nature est tournée vers le monde finno-ougrien, explique que la participation finlandaise soit toujours assurée. Pour quelques écrivains passionnés, beaucoup d'autres participants sont chercheurs ou simplement acteurs du monde intellectuel. En Hongrie, les délégués sont souvent les mêmes: une partie de chercheurs (Péter Domokos est resté fidèle jusqu'au bout, Katalin Nagy fait depuis 1978 partie de la direction, Virág Dyekiss, qui représente, seule, la jeune génération a participé à plusieurs reprises), et une partie de gens de lettres, traducteurs et écrivains, caractérisés par leur appartenance à la classe d'âge des soixante ans et plus. C'est qu'en Hongrie, la parenté finno-ougrienne n'est pas toujours très bien vue et semble peu inspirer les jeunes générations. Ce qui ne veut pas dire qu'il ne serait pas possible d'associer plus de jeunes. En Estonie, au contraire, le nombre de chercheurs est réduit, mais Valton a réussi de manière générale à associer des écrivains et traducteurs jeunes, de la génération des trente ans, qui ont la curiosité tout autant de la Russie que du monde finno-ougrien, et dont beaucoup, mais pas tous, proviennent d'Estonie du Sud. D'ailleurs plusieurs d'entre eux ont eu des prix littéraires et sont connus dans le monde littéraire estonien, comme Kristiina Ehin ou Indrek Koff qui, respectivement depuis 2010 et 2013, font partie de la direction de l'association.

66 Ainsi, tout le monde y retrouve son compte, mais surtout les citoyens de la Fédération de Russie. L'intérêt pour ce type d'initiative ne peut venir que des écrivains proprement dits: les chercheurs, en effet, ont en général bien d'autres cadres et d'autres subventions pour aller rencontrer les interlocuteurs dont ils ont besoin.

67 Cet article avait pour objectif de développer une réflexion sur un processus en cours, mais à propos duquel il n'a été écrit que fort peu. J'espère qu'il suscitera la discussion et que d'autres visions que la mienne en ressortiront, enrichissant ainsi la connaissance que nous pouvons avoir d'un exemple intéressant de travail en réseau dans le monde finno-ougrien. 


\section{BIBLIOGRAPHIE}

COLARZ Walter, 1956, Die Nationalitätpolitik der Sovjetunion, Frankfurt am Main.

GRIŠKINA, 1994, ГРИШКИНА, МАРГАРИТА ВЛАДИМИРОВНА, УДМУРТИЯ В ЭПОХУ ФЕОДАЛИЗМА (КОНЕЦ

XV - ПЕРВАЯ ПОЛОВИНА ХІХ В.), ИЖЕВСК.

HÕIMUSIDEMED, 1997, http://www.suri.ee/hs/, recueil d'articles non paru sur support papier, Fenno-ugria.

IL'MINSKIJ, 1898, ИЛЬМИНСКИЙ, НИКОЛАЙ ИВАНОВИЧ, ПИСЬМА Н.И. ИЛЬМИНСКОГО (К ОБЕРПРОКУРОРУ СВЯТЕЙШЕГО СИНОДА КОНСТАНТИНУ ПЕТРОВИЧУ ПОБЕДОНОСЦЕВУ), КАЗАНЬ.

ISTORIJA, 1987, I = ИСТОРИЯ УДМУРТСКОЙ СОВЕТСКОЙ ЛИТЕРАТУРЫ. ТОМ I, УСТИНОВ.

KAPPELER Andreas, 1982, Russlands erste Nationalitäten. Das Zarenreich und die Völker der Mittleren Wolga vom 16. bis 19. Jahrhundert, Köln-Wien.

КUVŠINOVA, 1990, КУВШИНОВа, РОЗА АНДРЕЕВНА, « ГОРЕЧЬ И РАДОСТЬ », КУЗЕБАЙ ГЕРД И

УДМУРТСКАЯ КУЛЬТУРА, ИЖЕВСК, 1990, С. 178-181

KUZNECOV, 1994, КУЗНЕЦОВ, НИКОЛАЙ СПИРИДОНОВИЧ, ИЗ МРАКА, ИЖеwsk.

MARIST, 1992, ИСТОРИЯ МАРИЙСКОГО КРАЯ, ЙОШКАР-ОЛА.

MEGYEK, 1993, Megyek élő testvéremhez, Finnugor költő́k antológiája. Szerk.: Katona Erzsébet, Budapest. NOLDE Boris, 1953 -La formation de l'empire russe : études, notes et documents 1-2, Paris.

PROZES, 1997, « Hõimuliikumine kahe maailmasõja ajal » http://www.suri.ee/hs/prozes (consulté le 10.02.2012)

REVOLUCIJA, 1930, РЕВОЛЮЦИЯ И НАЦИОНАЛЬНЫЙ ВОПРОС Т. III, МОСКВА.

TOULOUZE Eva, CAGNOLI Sébastien, LORENZINI Vincent, 2008, « Le $10^{\mathrm{e}}$ Congrès des écrivains finnoougriens à Joškar Ola », Études finno-ougriennes n 40, p. 240-245.

TOUlouze Eva, 1991, «Compte rendu de la première conférence des écrivains finno-ougriens (Joshkar-Ola, mai 1989) », Études finno-ougriennes $\mathrm{n}^{\circ}$ 23, p. 153-164.

Toulouze Eva, 2001, « Les peuples ouraliens de Russie et la culture de l'écrit », Hommage à Jean Perrot, Paris, p. 449-464.

TOULouzE Eva, 2006, « Le danger finno-ougrien en Russie (1928-1932) : les signes avant-coureurs des répressions staliniennes ", Études finno-ougriennes, n 38, p. 7 - 56.

TOUlouzE Eva, 2010, «Qui sont les Komis? Présentation générale », Les Komis, questions d'histoire et de culture, éds. Eva Toulouze \& Sébastien Cagnoli, Paris, p. 20-46.

\section{NOTES}

1. Le statut de Speranskij pour les peuples du Nord et de Sibérie est dès 1822 un exemple de l'émergence de cette catégorisation.

2. Jusqu'au début du $x x^{e}$ siècle. Dans le contexte de l'émergence des identités nationales, en effet, le mythe d'un ancien État fait son apparition : le cas le plus clair est 
celui des Komis, avec la Biarmija, fixée par Kallistrat Žakov (1916). Les Oudmourtes ont construit un mythe historique autour d'Idnakar. Mais parler d'ethnicité serait être anachronique pour ce qui est de ces époques reculées.

3. En mari : МАРИЙ КАЛЫК НЕРГЕН

4. En mari : МАРЛА КАЛЕНДАРЬ

5. C'est l'idée du Comité du Nord, cette institution mise en place par le pouvoir pour la gestion des zones boréales, et comportant nombre d'ethnographes et de personnes réellement compétentes, œuvrant dans l'intérêt des communautés (1924-1934).

6. Il suffit de dire que Konstantin Päts était en Estonie à l'initiative de la création de l'institution «Fenno-ugria », chargée de coordonner les activités pan-finno-ougriennes (Prozes 1997).

7. En finnois : M-A Castrénin seura.

8. Jeremei Aipin, Jumalaema verisel lumel, Kirjastuskeskus 2008.

9. Juri Vella, Järvetuul, Varrak 2011.

10. Vagatova, Maria: A kis tundrai ember. Ford.: Nagy Katalin. Larus, Budapest, 1998.

11. Tatjana Moldanova, A Malangi Anna középső világ-beli élete. A civilizáció érintése, Budapest 2000.

12. Szvetlana Gyiniszlamova: Vízcsepp. Manysi versek., Szerk. Sipőcz Katalin. Jegyzetek: Nagy Katalin és Sipőcz Katalin. Szegedi Egyetemi Kiadó, Juhász Gyula Felsőoktatási Kiadó. Szeged, p. 1-91

13. Nikolai Abramov: Kahtisti koumnekümne koume - Kétszer harminchárom, Válogatott versek vepsze és magyar nyelven. Válogatta és fordította Nagy Katalin. A Finnugor Népek Világkongresszusa, Budapest, 172 p.

14. Jurij Vella, Féhér kiáltások, Minoritates mundi, Szombathely 2004.

15. ПЧЕЛОВОДОВА Н.А., «КНИГИ ВЗАИМНЫХ ПЕРЕВОДОВ ФИННО-УГОРСКОЙ лиТЕРАТУРЫ 1996-2006", инВожО, 2007, nº 5,6, c.48-53; Soome-ugri kirjandusi eesti keelde tõlkimise ajaloost.

http://www.fennougria.ee/index.php?id=21713\&highlight=fenno

16. Sergei Tšavain, Elnet, suomentanut Arto Moisio, Faros-kustannus 2008.

17. Il suffit de rappeler le succès de l'ouvrage de Nils-Aslak Valkeapää Beaivi áhčážan, 1988.

18. Lequel a démissionné au bout d'un mandat. En 2013, c'est le Hongrois János Pusztay qui a été élu à cette fonction.

19. Depuis la rédaction de cet article, un Congrès a été organisé en novembre 2013 à Salehard, dans le Jamal. Le prochain, prévu en 2015, devrait avoir lieu en Hongrie.

20. Par exemple le Mari des collines Valerij Alikov.

21. Par exemple le Komi Nikolaj Kuznecov.

22. Par exemple l'Oudmourte Muš Nadi.

23. Par exemple l'Oudmourte Elena Rodionova.

24. J'ai personnellement participé à tous les congrès sauf le premier et celui de Petrozavodsk. Au congrès de Joškar Ola, j'étais accompagnée de Sébastien Cagnoli et Vincent Lorenzini. Au Congrès d'Oulu, Marie Casen s'est jointe à ce groupe.; cf. 
Toulouze, Cagnoli, Lorenzini 2008 ; au congrès de Salehard, le groupe était composé de Sébastien Cagnoli, Catherine Le Roux, Dominique Samson et moi-même.

\section{RÉSUMÉS}

Répondant à l'appel de Miklaj Rybakov, écrivain et leader du mouvement mari, des écrivains de toutes les régions finno-ougriennes rassemblèrent à Joškar Ola en 1989 et purent alors pour la première fois formuler librement toutes leurs inquiétudes sur la position minorée des langues finno-ougriennes en Russie. Depuis, ils ont poursuivi cette tradition, se sont constitués en 1993 en Association et se rencontrent tous les deux ou trois ans dans l'une des régions finnoougriennes d'Eurasie. Si les premières années les écrivains ont profité de ce forum pour exposer leurs difficultés, à partir de 1996 ils se sont tournés vers des tâches plus pratiques et constructives, c'est-à-dire vers la traduction mutuelle et l'étude des différentes branches prioritaires de la littérature. C'est cette histoire et ces évolutions que retrace cet article.

Finno-Ugric identity has played a central role in Komi society since the $19^{\text {th }}$ century national awakening. It is based on a double idea: on the one hand, on the supposed common origin of peoples speaking Finno-Ugric languages; on the other hand, on belonging to a contemporary international community of peoples who are supposed to share some part of their culture because of this common origin.

From this starting point, this article's aim is to assess the position of Komi literature within the Finno-Ugric community: what kind of relations can we identify between Komi literature and other Finno-Ugric literatures? What are the characteristics that testify to a common heritage or to recent mutual influence? Finally, what are these characteristics both in form and in contents?

Soome-ugri identiteet on mänginud komi ühiskonnas keskset rolli alates nende rahvuslikust ärkamisest 19. sajandil. See põhineb kahel seisukohal: esiteks on soome-ugri rahvad oletatavasti ühtse päritoluga ning teiseks on komid osa kaasaegsest rahvusvahelisest kogukonnast, kuhu kuuluvate rahvaste kultuur peaks eelmainitud ühise päritolu tõttu osaliselt kattuma.

Artikli eesmärk on hinnata antud lähtepunktist komi kirjanduse paiknemist soome-ugri kogukonnas: millised on komi ja muu soome-ugri kirjanduse vahelised seosed? Millised tunnusjooned osutavad ühisele pärandile või hiljutisele vastasmõjule? Ja lõpetuseks, millised on nimetatud tunnusjooned vormilt ja sisult? 


\section{INDEX}

disciplines français, hongrois, allemand, mari, russe, tatar, finnois, estonien, anglais nomsmotscles Estoniens, Finlandais, Khantys, Komis, Lettons, Mansis, Maris, Maris des collines, Maris des plaines, Mordves, Nénetses, Nénetses de la toundra, Nénetses des forêts, Oudmourtes, peuples du Nord, Polonais, Russes, Tatars, Ukrainiens

Thèmes : littérature

Mots-clés : culture écrite, identité, intellectuels, traductions, vie littéraire

Keywords : identity, intellectuals, translations, Written culture, literary life, end of the 20th century, beginning of the 21st century, Arctic peoples, Estonians, Finns, Forest Nenets, Hill Mari, Khanty, Komi, Mansi, Mari, Meadow Mari, Mordvinians, Nenets, Poles, Russians, Tatars, Tundra Nenets, Udmurt, Ukrainians

Index géographique : Komi-permiak (district), Espoo, Oulu, Eger, Islande, Joškar-Ola, Jamal (raïon), Morko, Idnakar, Nadym, Petrozavodsk, Salehard, Saransk, Syktyvkar, Hanty-Mansijsk, Votkinsk, Nénetses (district autonome), Iževsk

Index chronologique : XXe siècle (fin), XXe siècle, XXIe siècle 